\title{
Counseling of Patients with Severe Psychiatric Conditions and Referral to Smoking Cessations Programs
}

\author{
Mariwan Husni, Mazin Burhan, and Mohamed Mazin
}

\section{ABSTRACT}

Background: Cigarette smoking and Nicotine dependence are highly prevalent in patients with severe and chronic mental illness. Psychiatric patients consume more cigarettes than general population. Smokers with chronic mental illness can quit smoking with appropriate intervention by smoking cessations programs. These patients can benefit from pharmacotherapy, but consideration should be given for possible interaction with patients' psychiatric medications. Tobacco use and dependence need to be formally documents on electronic medical records and be offered a referral to smoking cessations programs.

Method: All patients in a community rehabilitation service ward in Northwest London were interviewed to identify the type, quantity, and duration of their tobacco. Their electronic clinical records were reviewed to confirm their age, ICD 10 Primary Diagnosis, ICD10 Tobacco dependence diagnosis, medications they take, record of ECG tests and presence of cardiopulmonary problems. Then their records were checked if they were given advice regarding their smoking habit, desire to quit smoking and agreement to be referred to a smoking cessation program.

Results and Discussion: Half of the total number of patients in the community psychiatric rehabilitation were cigarette smokers. Majority of the smokers had history of polysubstance abuse. Recording of current smoking status were not regularly update. Patients who had recorded status of being a smoker were all referred to a smoking cessation program and majority had counseling by the healthcare professionals in their unit. Attending the smoking cessation program in those patients have as good outcomes as general population.

Conclusions: A substantial number of patients in psychiatric rehabilitation units smoke cigarettes. If their smoking status is accurately documented, they would be referred to a formal smoking cessation program.

Keywords: Cigarette Smoking, Psychiatric Rehabilitation, Smoking Counselling, Recording Smoking Status.
Published Online: July 28, 2021

ISSN: $2736-5476$

DOI: 10.24018 /ejclinicmed.2021.2.3.102

\section{Husni*}

College of Medicine and Medical Sciences, Arabian Gulf University, Kingdom of Bahrain

Northern Ontario School of Medicine, NOSM, Ontario, Canada.

CNWL NHS Foundation Trust, London, UK.

(e-mail: mariwanh@agu.edu.bh)

M. Burhan

CNWL NHS Foundation Trust, London,

UK.

(e-mail: mazin.burhan@nhs.net)

M. Mazin

University of Nottingham, UK

(e-mail: mohamed.mazin@live.com)

*Corresponding Author

\section{INTRODUCTION}

Nicotine dependence is highly prevalent among patients with psychiatric disorders compared to general population [1]. Smokers with mental health problems smoke more heavily, are more nicotine-dependent and have smoked for longer than smokers in the general population [2]. As a group, smokers with psychiatric disorders consume a disproportionate number of cigarettes [3]. Nicotine intake per cigarette is higher in patients with chronic mental illness, which contributes to high serum nicotine levels in these patients compared to control without mental illnesses. The high nicotine levels result in dependence and withdrawal symptoms, even with moderate amounts of smoking [4].
The national Commissioning for Quality and Innovation (CQUIN) for hospitals in the UK, has given incentives to screen for tobacco use and offering National health service (NHS)-funded tobacco treatment to all inpatients to assist providers to implement [5]. The National Institute for Health and Care Excellence (NICE) guidance on reducing smoking in acute and mental health settings such screening and support need to be optimized.

There is need to develop and implement smoking cessation interventions in clinical settings such as inpatient rehabilitation wards for patients with severe and chronic mental illnesses. A survey of NHS staff revealed that mental healthcare professionals are significantly less positive about taking a role in supporting service users to quit smoking than 
their general healthcare colleagues [6], although mental health patients are as a likely to want quit smoking and they can do so with appropriate support as general population [7]. People with mental health problems need good access to services aimed at improving health including stop smoking services.

Health care professionals should always remember the importance of promoting healthy lifestyles and should encourage service users to consider the action they can take to promote their own physical wellbeing. Staff should raise service users' awareness about smoking cessation.

Given the growing body of evidence showing that quitting smoking is typically not detrimental to psychiatric symptoms and, in fact, may lead to improvements in mental health and wellbeing [8]. Nonetheless, people with a mental illness should be offered the same smoking cessation interventions that have been shown to be effective in the general population, with optimal treatment comprising behavioural and pharmacotherapy interventions, alone or in combination [9]. Smokers with chronic mental illness can successfully quit with standard cessation approaches, and longer maintenance on pharmacotherapy can reduce the typically high rates of relapse without detrimental effects on psychiatric symptoms [10]. An important consideration in treating nicotine dependence is the impact of smoking on psychiatric treatment. Smoking complicates the treatment and course of psychiatric disorders through its profound effect on the metabolism of pharmacotherapies and is therefore one of the factors that leads to individual differences in drug responses [11]. For example, smoking can interfere with the medications taken for schizophrenia and depression, therefore the doses of some psychotropic medications may need to be decreased following cessation [12].

Patients with mental illness can be offered the same cessation medications as the general population but should be monitored closely [13].

Progress in the development of cessation treatments for people with mental health problems has traditionally been slow, in part because smokers with a current mental disorder have been excluded from most smoking cessation trials [14].

Our study aimed to find out prevalence characteristics of smoking amongst inpatients in a community rehabilitation service ward. We also wanted to examine whether tobacco dependence was formally registered as a concurrent ICD 10 diagnoses on patients' records. The study also assessed if patients have been formally advised regarding their smoking and whether they were referred to a smoking cessation programme.

\section{METHOD}

All patients admitted to an inpatient community rehabilitation service ward in Northwest London were interviewed to find out the type, quantity, and duration of their tobacco use (such as cigarette smoking). Their electronic clinical records were reviewed to confirm their gender, age, ICD 10 Primary Diagnosis, ICD10 Tobacco dependence diagnosis, medications, record of ECG and presence of Cardiopulmonary problems. Then their records were checked if they were given Advice regarding their smoking habit, desire to quit smoking and referral to a smoking cessation program.

\section{RESUlTS AND DISCUSSION}

A total of 12 patients were admitted to the inpatient community rehabilitation service ward. Of those patients 2 $(17 \%)$ were females and $10(83 \%)$ were males.

The diagnosis of patients were schizophrenia 7 patients, Schizoaffective Disorder 2 patients, Bipolar Affective Disorder 2 patients, and Unspecified Pervasive Developmental Disorder 1 patient. The duration of smoking was ranging from 1 to 30 years.

The number of patients who were currently smokers or non-smokers was evenly divided (50\% in either group). There were discrepancies between what the patients were reporting as their current smoking state and what was recorded on their recorded. Two patients reported that they were smokers, but their smoking status (ICD 10 diagnosis of smoking) was not recorded, and two patients denied being currently smokers, were recorded (having an ICD 10 diagnosis) of being smokers. Four patients (three smokers and one nonsmoker) in the study had history of illicit drug abuse.

All the patients were on psychiatric medications and three of them were also on medications for physical health problems. One patient had a Cardiopulmonary problem. All patients except one had an ECG don during in the last 12 months.

Four of the six smokers in the study were given advice regarding smoking and they were also referred to a smoking cessation program. However, one of them did not accept to be referred.

Tobacco use is common in psychiatric patients and $50 \%$ of patients in the study were tobacco smokers. This is slightly higher than reported figures of the current prevalence of smoking $(41 \%)$ in patients with past-month mental illness [15].

The overwhelming majority of the patients had diagnosis of schizophrenia and related disorders. These diagnoses proportionately smoke more compared to patients having other psychiatric diagnosis. Recording the current smoking status on the patient records are not accurate. In our study a third of patients had inaccurate recording of the smoking status as an ICD 10 concurrent diagnosis. It is important to record the diagnosis of tobacco use accurately and keep it up to. Once the smoking status is established, psychiatric patients who smoke need to be offered help and guidance including referral to a smoking cessation program. It is reported that psychiatric patients have similar success rates to general population of stopping smoking.

History of polysubstance abuse is higher in smokers versus nonsmokers. Those with positive history of polysubstance in the study, $75 \%$ were cigarette smokers.

Smoking contributes to physical health problems, and they need regular monitoring. All patients except one had an ECG done in the last year, which is a good rate but since these patients were in a rehabilitation setting in the community, that is closely related and accessible to a hospital, monitoring of every patient would be expected. Cardiopulmonary illness was present in only one patient, which is lower than expected in this population. 
Two thirds of patients who smoked were given advice regarding smoking and all of them were offered a referral to a smoking cessation program. However, all patients particularly those who are in rehabilitation due to chronic and severe mental illness, should be given advice regarding smoking and its harmful effect on the health, and all who smoke should be advised and referred to smoking cessation programs. Like general population patients with severe mental illness would benefit from smoking cessation programs with good outcomes.

\section{CONCLUSION}

Cigarette smoking and Nicotine dependence are highly prevalent in patients with severe and chronic mental illness. Current smoking status must be accurately documented in patients going through rehabilitation for severe psychiatric conditions. Appropriate counseling regarding smoking and referral to smoking cessations programs will benefit these patients.

\section{REFERENCES}

[1] Vanable PA, Carey MP, Carey KB, Maisto SA. Smoking among psychiatric outpatients: relationship to substance use, diagnosis, and illness severity. Psychol Addict Behav. 2003 Dec; 17(4):259-65.

[2] Kumari, V. \& Postma, P. (2005) Nicotine use in schizophrenia: the self medication hypothesis. Neuroscience and Biobehavioural Reviews, 29, 1021-1034.

[3] Grant BF, Hasin DS, Chou SP, Stinson FS, Dawson DA. Nicotine dependence and psychiatric disorders in the United States: results from the national epidemiologic survey on alcohol and related conditions. Archives of General Psychiatry. 2004;61(11):1107-1115.

[4] Williams JM, Ziedonis DM, Abanyie F, et al. Increased nicotine and cotinine levels in smokers with schizophrenia and schizoaffective disorder is not a metabolic effect. Schizophr Res 2005; 79:323-35.

[5] NHS England. Commissioning for Quality and Innovation (CQUIN). NHS England, 2016 (https://www.england.nhs.uk/wpcontent/uploads/2016/03/cquin-guidance-16-17-v3.pdf).Google Scholar.

[6] McNally L, Oyefeso A, Annan J, Perryman K, Bloor R, Freeman S, Wain B, Andrews H, Grimmer M, Crisp A, Oyebode D, Ghodse H. A survey of staff attitudes to smoking-related policy and intervention in psychiatric and general health care settings. J Public Health. 2006; 28:192-196.

[7] Hall SM, Prochaska JJ. Treatment of smokers with co-occurring disorders: emphasis on integration in mental health and addiction treatment settings. Апnи Rev Clin Psychol 2009; 5:409-31.

[8] Taylor G, McNeill A, Girling A, Farley A, Lindson-Hawley N, Aveyard $\mathrm{P}$ et al. Change in mental health after smoking cessation: systematic review and meta-analysis BMJ 2014; 348: g1151.
[9] Patnode CD, Henderson JT, Thompson JH, Senger CA, Fortmann SP, et al. Behavioral counseling and pharmacotherapy interventions for tobacco cessation in adults, including pregnant women: A review of reviews for the US Preventive Services Task Force. Annals of Internal Medicine, 2015. Available from: http://www.ncbi.nlm.nih.gov/pubmed/26389650.

[10] Tidey JW and Miller ME. Smoking cessation and reduction in people with chronic mental illness. British Medical Journal, 2015; 351:h4065. Available from: http://www.ncbi.nlm.nih.gov/pubmed/26391240.

[11] Minichino A, Bersani FS, Calo WK, Spagnoli F, Francesconi M, et al. Smoking behaviour and mental health disorders--mutual influences and implications for therapy. International Journal of Environmental Research and Public Health, 2013; 10(10):4790-811. Available from: http://www.ncbi.nlm.nih.gov/pubmed/24157506.

[12] Oliveira P, Ribeiro J, Donato H, and Madeira N. Smoking and antidepressants pharmacokinetics: A systematic review. Annals of General Psychiatry, 2017; 16:17. Available from: http://www.ncbi.nlm.nih.gov/pubmed/28286537.

[13] Anthenelli RM, Benowitz NL, West R, St Aubin L, McRae T, et al. Neuropsychiatric safety and efficacy of varenicline, bupropion, and nicotine patch in smokers with and without psychiatric disorders (EAGLES): A double-blind, randomised, placebo-controlled clinical trial. Lancet, 2016. Available from: http://www.ncbi.nlm.nih.gov/pubmed/27116918.

[14] Hitsman B, Moss T, Montoya I, and George T. Treatment of tobacco dependence in mental health and addictive disorders. Canadian Journal of Psychiatry, 2009; 54(6):368-78. Available from: http://www.ncbi.nlm.nih.gov/pubmed/19527557.

[15] Lasser K, Boyd JW, Woolhandler S, Himmelstein DU, McCormick D, Bor DH. Smoking and mental illness: A population-based prevalence study. JAMA. 2000;284(20):2606-2610.

M. Husni is professor and chair of the department of psychiatry at the Arabian Gulf University, Kingdom of Bahrain. He is also a professorial faculty at the Northern Ontario School of Medicine (NOSM), Ontario, Canada. He is a fellow of the Royal College of Physicians and Surgeons in Canada. He is also a fellow of the Royal College of Psychiatrists United Kingdom

He has completed his residency at the University of Western Ontario, Canada. He also has completed a fellowship in addictions psychiatry at the University of Western Ontario. He holds a PGC in Cognitive Analytic Therapy. He is currently the Chairperson of Psychiatry at the AGU, Manama Kingdom of Bahrain. He is also a Consultant Psychiatrist at the King Hamad University Hospital (KHUH), Busaiteen, Kingdom of Bahrain. He is a consultant psychiatrist at the CNWL, London, United Kingdom.

Professor Husni is the Chair of Personality Disorder, Arab Psychiatry Federations. He is a member of the College of Physicians and Surgeons of Ontario, Canada. He is a member of the American Psychiatric Association.

Dr. Mazin Burhan is a specialty doctor with the Central and Northwest (CNWL) NHS Foundation Trust, London, UK. He has M. SC. in Cognitive Behavioral Therapy (CBT). He is Approved under section (12) of mental health act, United Kingdom.

Mr. M Mazin is a $4^{\text {th }}$ year medical student, University of Nottingham, Nottingham, United Kingdom. 